1992 General Election. After the announcement of a Conservative victory rates fell, with three cases presenting during this post-election weekend compared with a weekend average of $13(99 \%$ confidence limits; 3.53-22.08). Masterson \& Platt (1989) have previously demonstrated an association between British general elections and parasuicide. In a traditionally Labour voting area, the victories of a Conservative Government in 1970, 1979, 1983 and 1987 were met with an increased rate of parasuicide, while the rate fell following the election of a Labour Government in 1974.

In 1992 the country re-elected a Conservative Government. Nottingham had strongly supported Labour. The result was presumably disappointing for the majority, and in contrast to the findings of Masterton \& Platt there were fewer cases of DSP. The two observations may not be comparable because the 'effects' were demonstrated over different time scales and Masterton \& Platt made no comment on immediate effects.

We have speculated as to the reasons behind the Nottingham phenomenon. We wonder if the fall in our rates of DSP reflects relief that at long last the electioneering was over. I would be interested to hear whether other centres observed similar short-term changes in presentations of self-poisoning cases following the 1992 General Election.

Masterton, G. \& Platt, S. (1989) Parasuicide and general elections. British Medical Journal, 298, 803-804.

Department of Psychiatry

MARK Steels

University Hospital

Queen's Medical Centre

Nottingham NG7 2UH

\section{Tattoed female psychiatric patients}

SIR: While tattooing among women is becomingly increasingly fashionable (Mercer \& Davies, 1991) there is little research on the topic. Females in Borstal who were heavily tattooed were more criminal and aggressive in their attitudes and behaviour compared with less heavily tattooed or non-tattooed inmates (Taylor, 1968) but there is no research on female psychiatric patients. It is known that tattooed male psychiatric patients are more likely to have a diagnosis of personality disorder (Gittleson et al, 1969) and an association has also been found with men who harm themselves (Virkkunen, 1976). We examined female acute psychiatric in-patients and day patients in Sheffield. Four had tattoos and all agreed to be interviewed.
Case reports. Patient $\mathrm{A}$ was a 21-year-old with a diagnosis of borderline personality disorder and a history of repeated deliberate self-harm. She had a professional tattoo at the top of her right arm which had been carried out when she was intoxicated in the company of friends at the age of 17. The tattoo was of a panther head baring its teeth. She disliked the tattoo which she wanted to have removed, and kept it covered most of the time. She had been sexually abused from the age of 3 to 6 by her father and stepbrother.

Patient $B$ was a 43-year-old with a diagnosis of borderline personality disorder, alcohol dependency and major affective disorder. She had had multiple admissions with depressive episodes, for detoxification, and following episodes of deliberate self-harm. She had four tattoos, two professional and two that she had done herself. All four were done in her early 20 s while intoxicated with friends and were on her forearms. She had a mixed reaction to the tattoos. She disliked the two professional tattoos, one which was a heart with an arrow through it and the other which coupled her name with that of a former patient. However the two amateur tattoos which were of her mother's name and the names of her three sons gave her comfort at times. She gave a history of being sexually abused between the ages of 5 and 15 by an uncle.

Patient $C$ was a 38-year-old with a diagnosis of borderline personality disorder. She had a long history of repeated deliberate self-harm. She had had two tattoos, one of a pin saint on the right upper arm and one of a man's name on the left lower arm. They were amateur tattoos done by her peers when she was 12 years old. She was ashamed of them and had them removed at the age of 18 leaving a visible scar. She gave a history of having been sexually abused by her father from the age of 10 until he had died when she was 35 .

Patient D was a 27-year-old with a diagnosis of borderline personality disorder and alcohol dependency. She had a history of self-mutilation. She had three amateur tattoos of male names on her forearms done by herself at the age of 17 while intoxicated in the company of friends. She now expresses indifference towards the tattoos. She gave a history of sexual abuse between the ages of 6 and 12 by her stepfather.

Although sexual abuse is common among psychiatric patients it is interesting that all four tattooed patients had been sexually abused. There are several explanations for tattooing. One is the concept of the 'exoskeletal defence' (Popplestone, 1963) suggesting that people with tattoos expect to be attacked and therefore use tattoos as a stereotyped display or symbol of physical strength and psychological aggressiveness. Another concept is that tattoos and other forms of self-mutilation are a way of showing a negative attitude and violence towards oneself. Both explanations could apply to sexual abuse victims. More research needs to be undertaken on a larger controlled sample to investigate whether a correlation exists. Mercer \& Davies (1991) posed the question of whether tattoo removal should be provided by a cash-limited health service. If tattoos 
are a stigma of abuse then we feel that their removal should be readily available.

Gittleson, N. L., Wallen, G. D. P. \& Dawson-Butterworth, K. (1969) The tattooed psychiatric patient. British Journal of Psychiatry, 115, 1249-1253.

MerCer, N. S. G. \& DA vies, D. M. (1991) Tattoos. British Medical Journal, 303, 38.

Popplestone, J. A. (1963) A syllabus of the exoskeletal defences. Psychological Record, 13, 15-25.

TAYLOR, A. J. W. (1968) A search among borstal girls for the psychological and social significance of tattoos. British Journal of Criminology. 8, 170-185.

VIRKKUNEN, M. (1976) Self mutilation in antisocial personality (disorder). Acta Psychiatrica Scandinavia, 54, 347-352.

HEATHER INCH RHODRI HUWS

Department of Psychiatry

Whiteley Wood Clinic

Woodfindin Road

Sheffield S10 3TL

\section{Simple schizophrenia - a forgotten diagnosis}

SIR: Vostanis \& Dean (Journal, August 1992, 161, 265-267) reported two adult patients with selfneglect and found them interesting for the following reasons: (a) onset in young adulthood (age 35 and 38 at referral) rather than old age; (b) no psychiatric diagnosis was possible in the DSM-III-R in view of "the long history of generally stable behaviour and no confirmed psychotic symptoms" which precluded schizophrenia; (c) depot neuroleptic was ineffective and patients declined psychiatric treatment.

In our view, the case histories are typical of 'simple schizophrenia', an uncommon condition first described by Eugen Bleuler, later accepted by Emil Kraepelin, and recently reviewed by Black \& Boffeli (1989). Although ICD-9 advised that the diagnosis should be made 'sparingly', it is retained (F20.6) in ICD-10 because of its "continued use in some countries'. The essence of this condition is insidious psychosocial deterioration without obvious psychotic symptoms. Associated features include neglect of hygiene, social isolation, loss of initiative, oddities of conduct, hoarding useless items, vague digressive speech, and overvalued ideas. As the case histories provided by Vostanis \& Dean include virtually all the above features, we do not agree that no psychiatric diagnosis is possible. Further, as few positive symptoms were present, the relative lack of response to neuroleptic treatment is expected. Even though the DSM-III-R deleted simple schizophrenia and has attempted to fill the vacuum by creating the entity of schizotypal personality disorder, there is conceptual problem in applying the latter diagnosis to the two cases because of the marked avolition, functional deterioration and self-neglect.

Simple schizophrenia remains of heuristic interest and a clinically useful differential of self-neglect in young adulthood. Bleuler suggested that outside of hospitals, it might be as common as other forms of schizophrenia (Black \& Boffeli, 1989). More research on its validity is warranted.

BLACK, D. W. \& Bofreu, T. J. (1989) Simple schizophrenia: past, present, and future. American Journal of Psychiatry, 146, 1267-1273.

Department of Psychiatry

11/F Prince of Wales Hospital

Shatin

Hong Kong

\section{Phenomenology and schizophrenia}

SIR: I read Dr Mortimer's article on phenomenology in schizophrenia research with enjoyment (Journal, September 1992, 161, 293-297). Her three-level model of schizophrenia covers the fact that natural phenomena can be observed on multiple levels, providing a framework for understanding complex causes of disease that are not simple 'lesions' on any one level.

She omitted, however, any level relating to 'mind', or intersubjective reality, the organisational level at which schizophrenia most clearly manifests as a problem. For example, by wishing good morning to a person with schizophrenia, that something is radically wrong is apparent; and, as she points out, such information is at least as reliable as sophisticated instrumentation.

Models of mind are often said to be 'unscientific', because they are difficult to test in traditionally accepted ways: complex philosophical questions are raised, which by their nature may have to remain unresolved. Yet in practice, it seems to be assumed that they have been resolved. The concept of mind is largely ignored by modern psychiatry, which appears mistrustful of abstract ideas.

It was, perhaps, the tendency of the psychoanalytical establishment to reject insights and advances from outside its own world view, particularly the revolutionary (and serendipitous) pharmacological discoveries in the mid-20th century, that led to the decline in the influence of its ideas. However, as usual, a new orthodoxy simply took the place of the old. The historical perspective allows us now to see 